$1-2003$

\title{
Clinical Blood Flow Quantification with Segmented k-Space Magnetic Resonance Phase Velocity Mapping
}

\author{
George P. Chatzimavroudis \\ Cleveland State University \\ Haosen Zhang \\ EdflkedathdsStatedtriktersity works at: https://engagedscholarship.csuohio.edu/encbe_facpub

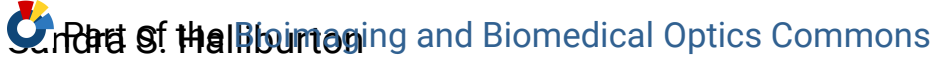 \\ Alovelabedsclicicesoundatianwork benefit you? Let us know!

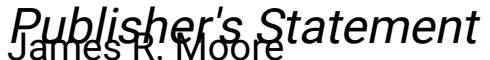

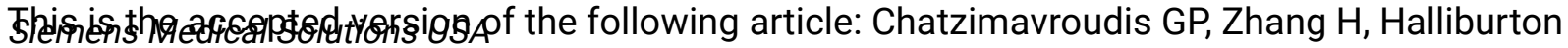

SS, Moore JR, Simonetti OP, Schvartzman PR, Stillman AE, White RD. Clinical blood flow Orlande Simonetti
guantification with segmented k-space magnetic resonance phase velocity mapping. Journal of
Siemens Medical Solutions USA

Magnetic Resonance Imaging. 2003;17:65-71., which has been published in final form at

http://onlinelibrary.wiley.com/doi/10.1002/jmri.10231/abstract

See next page for additional authors

\section{Original Citation}

Chatzimavroudis GP, Zhang H, Halliburton SS, Moore JR, Simonetti OP, Schvartzman PR, Stillman AE, White RD. Clinical blood flow quantification with segmented k-space magnetic resonance phase velocity mapping. Journal of Magnetic Resonance Imaging. 2003;17:65-71.

\section{Repository Citation}

Chatzimavroudis, George P.; Zhang, Haosen; Halliburton, Sandra S.; Moore, James R.; Simonetti, Orlando; Schvartzman, Paulo R.; Stillman, Arthur E.; and White, Richard D., "Clinical Blood Flow Quantification with Segmented k-Space Magnetic Resonance Phase Velocity Mapping" (2003). Chemical \& Biomedical Engineering Faculty Publications. 108.

https://engagedscholarship.csuohio.edu/encbe_facpub/108

This Article is brought to you for free and open access by the Chemical \& Biomedical Engineering Department at EngagedScholarship@CSU. It has been accepted for inclusion in Chemical \& Biomedical Engineering Faculty Publications by an authorized administrator of EngagedScholarship@CSU. For more information, please contact library.es@csuohio.edu. 


\section{Authors}

George P. Chatzimavroudis, Haosen Zhang, Sandra S. Halliburton, James R. Moore, Orlando Simonetti, Paulo R. Schvartzman, Arthur E. Stillman, and Richard D. White 


\title{
Clinical Blood Flow Quantification With Segmented k-Space Magnetic Resonance Phase Velocity Mapping
}

\author{
George P. Chatzimavroudis, PhD, ${ }^{1,2 *}$ Haosen Zhang, MS, ${ }^{2}$ Sandra S. Halliburton, $\mathrm{PhD},{ }^{1,2}$ \\ James R. Moore, PhD, ${ }^{3}$ Orlando P. Simonetti, PhD, ${ }^{3}$ Paulo R. Schvartzman, MD, ${ }^{1}$ \\ Arthur E. Stillman, MD, ${ }^{1}$ and Richard D. White, $\mathrm{MD}^{1}$
}

\begin{abstract}
Purpose: To evaluate the accuracy of segmented k-space magnetic resonance phase velocity mapping (PVM) in quantifying aortic blood flow from through-plane velocity measurements.

Materials and Methods: Two segmented PVM schemes were evaluated, one with seven lines per segment (seg-7) and one with nine lines per segment (seg-9), in twenty patients with cardiovascular disease. A non-segmented (non-seg) PVM acquisition was also performed to provide the reference data.

Results: There was agreement between the aortic flow curves acquired with segmented and non-segmented PVM. The calculated systolic and total flow volume per cycle from the seg-7 and the seg- 9 scans correlated and agreed with the flow volumes from the non-seg scans (differences $<5 \%$ ). Sign tests showed that there were no statistically significant differences $(P$-values $<0.05)$ between the segmented and the non-segmented PVM measurements. Seg-9, which was the fastest among the three sequences, provided adequate spatial and temporal resolution ( $>10$ phases per cycle).
\end{abstract}

Conclusion: Segmented k-space PVM shows great clinical potential in blood flow quantification.

Key Words: magnetic resonance imaging; magnetic resonance phase velocity mapping; segmented k-space; blood flow; flow quantification; aorta; velocity

MAGNETIC RESONANCE (MR) phase velocity mapping (PVM) has been widely used clinically to measure blood

\footnotetext{
${ }^{1}$ Section of Cardiovascular Imaging, Division of Radiology, The Cleveland Clinic Foundation, Cleveland, Ohio.

${ }^{2}$ Laboratory of Biofluid Mechanics and Cardiovascular Imaging, Department of Chemical Engineering, Cleveland State University, Cleveland, Ohio.

${ }^{3}$ Magnetic Resonance Division, Siemens Medical Solutions USA, Chicago, Illinois.
}

velocity and flow. The technique is based on the principle that, by using the proper bipolar magnetic field gradients in the direction of interest, the velocity of the spins in that direction can be encoded within the phase of the detected signal (1). There have been many in vitro and in vivo studies evaluating PVM for flow quantification (2-17). For in vitro studies, the accuracy and precision of PVM was found to be consistently high, with errors lower than $10 \%(2-7)$. In vivo studies showed a good correlation between PVM and Doppler ultrasound or other flowmetric techniques $(2,4,8-11)$. PVM has been extensively used to visualize and quantify cardiac, arterial, and venous blood flow with high precision $(7,10-17)$.

Conventional PVM involves a non-segmented gradient-echo sequence with bipolar velocity-encoding gradients in the direction(s) of interest. The acquisition is prospectively or retrospectively ECG-gated to acquire a number of time-phases throughout the cardiac cycle. Only one line of k-space is acquired for each time-phase per cardiac cycle, resulting in a scanning time of several minutes (usually three to five minutes, depending on functional and imaging parameters) for acquisition of a single velocity direction in one imaging slice. Considering that PVM measurements constitute only part of a complete cardiac MR examination and that new diagnostic approaches have involved multi-slice and multidirectional velocity acquisitions $(6,18,19)$, this non-segmented, single k-space line scheme becomes less practical clinically.

With the development of rapid imaging sequences, such as turbo gradient echo (TGE) and echo planar, faster PVM has become possible (20-31). Instead of a single k-space line, a segment of $\mathrm{k}$-space lines are acquired per time-phase and cardiac cycle. As a result, the acquisition can be performed in seconds. Segmented TGE has shown good flow-behavior and can provide high temporal and spatial resolution acquisitions. It is, therefore, promising for reliable rapid flow quantification even in cases of moderately disturbed flow and vessel motion (25-31).

In a recent in vitro study (31), we showed that segmented k-space PVM provides accurate flow results under steady and pulsatile flow conditions (errors < 5\%). Segmented PVM measurements with five, seven, 
and nine k-space lines per segment agreed very closely with non-segmented measurements under identical experimental conditions. Therefore, the aim of the present study was to clinically evaluate segmented PVM as a blood flow quantification technique, by measuring blood flow in the human ascending aorta. Of interest was the effect of increasing the number of $\mathrm{k}$-space lines per segment on the accuracy of the results.

\section{MATERIALS AND METHODS}

MR PVM measurements were performed in the ascending aorta of twenty patients with cardiovascular disease (16 males, average age 61 years; four females, average age 73 years) using a 1.5 Tesla whole-body MRI scanner (Sonata, Siemens Medical Solutions, Erlangen, Germany) with a maximum gradient strength of 40 $\mathrm{mTesla} / \mathrm{m}$. Thirteen patients had aortic dilatation and aneurysms (six with aortic regurgitation) with mid-ascending aorta diameter 4.5-6.0 cm. Six patients had ischemic heart disease (two with mitral regurgitation, one with combined aortic stenosis/aortic regurgitation/mitral regurgitation). One patient had a left ventricular aneurysm. The study protocol was approved by the Institutional Review Board of the Cleveland Clinic Foundation.

Initial true-FISP (fast imaging with steady-state precession) scout images in all three spatial orientations (axial, coronal, and sagittal) showed the location of the ascending aorta. The through-plane velocity of blood was measured in an imaging slice placed perpendicular to the long-axis of the ascending aorta, at the level of pulmonary artery bifurcation. Three PVM acquisitions were performed, at the same location in each subject, using the following three sequences: (a) conventional non-segmented gradient echo (non-seg); (b) segmented TGE with seven $\mathrm{k}$-space lines per time-phase and cardiac cycle (seg-7); and (c) segmented TGE with nine lines per time-phase and cycle (seg-9).

The non-seg scans were 120-180 seconds long, whereas the segmented scans were 10-16 seconds long, depending on the heart rate. Each of the segmented k-space scans was performed during a breathhold. In all sequences, the flow-sensitive and the flowcompensated data were acquired in an interleaved manner in the same cycle. The flip angle was $30^{\circ}$. The slice thickness was $5 \mathrm{~mm}$. The scan percentage factor (percentage of $\mathrm{k}$-space filled) was $75 \%$ for the non-seg scans, $55 \%$ for the seg-7 scans, and 56\% for the seg-9 scans. The $75 \%$ rectangular field of view (FOV) was varied from $210 \times 280 \mathrm{~mm}^{2}$ to $263 \times 350 \mathrm{~mm}^{2}$, depending on patient size. Combination of the scan percentage factor with the rectangular FOV factor resulted in an acquired number of 144,105 , and $108 \mathrm{k}_{\mathrm{y}}$-lines for the non-seg, seg-7, and seg-9 scans, respectively. The nominal voxel size varied between $1.5 \times 1.1 \times 5.0 \mathrm{~mm}^{3}$ and $1.8 \times 1.4 \times 5.0 \mathrm{~mm}^{3}$ for sequence (a), between $2.0 \times 1.1 \times 5.0 \mathrm{~mm}^{3}$ and $2.5 \times 1.4 \times 5.0 \mathrm{~mm}^{3}$ for sequence (b), and between $1.9 \times 1.1 \times 5.0 \mathrm{~mm}^{3}$ and $2.4 \times 1.4 \times 5.0 \mathrm{~mm}^{3}$ for sequence (c). Therefore, for a reconstructed matrix of $256 \times 256$, the interpolated voxel size varied from $1.1 \times 1.1 \times 5.0 \mathrm{~mm}^{3}$ to $1.4 \times$ $1.4 \times 5.0 \mathrm{~mm}^{3}$ in all sequences.

The shortest (2.3-3.0 msec) allowable echo time (TE) was used for all measurements. All acquisitions were prospectively ECG-gated, resulting in 19-35, 13-25, and 11-21 time-phases per cycle for the non-seg, seg-7, and seg- 9 acquisitions, respectively. The temporal resolution of the segmented acquisitions was improved by using echo view-sharing (32). The resulting resolution was $30 \mathrm{msec}, 40-46 \mathrm{msec}$, and $50-57 \mathrm{msec}$ for the non-seg, seg-7, and seg-9 acquisitions, respectively. The time corresponding to each time-phase was the time of acquisition of the central line of the segment, since that line contained the most useful velocity information of the segment.

In the non-seg sequence, the repetition time (TR) was $15 \mathrm{msec}$. In seg-7 and seg-9, the time to acquire data for any one $\mathrm{k}_{\mathrm{y}}$-line (actual TR) varied between $5.2 \mathrm{msec}$ and $5.6 \mathrm{msec}$, depending on imaging parameters such as FOV. The echo was acquired asymmetrically within the data acquisition period. The peak of the echo occurred at data-point 82 of 256 and the missing data points were zero-filled. The velocity encoding value was 250 $\mathrm{cm} /$ second.

Each PVM acquisition produced a series of magnitude and phase images. All images were transferred to a work-station (Ultra-10, SUN Microsystems, Inc., Palo Alto, CA). A FORTRAN program was used to examine the images for the presence of any residual phase offsets $(5,33)$. When phase offsets were detected, they were removed based on a two-dimensional regression analysis model, and the phase values of the phase images were converted to velocity values ( $\mathrm{cm} / \mathrm{second})$, based on their proportionality. The images were further processed using Transform (Version 3.4, Research Systems, Inc., Boulder, $\mathrm{CO}$ ) to calculate the flow rates and flow volumes. The aortic cross-section was defined as the region of interest, and the velocity was integrated over the aortic cross-sectional area to determine the flow rate (mL/second) through the aorta for each timephase. Integration of the aortic flow curve over systole and over the entire cardiac cycle provided the systolic and total flow volumes (mL/cycle), respectively. When no phase offset was detected, the images were processed using manufacturer software (ARGUS Cardiac Analysis, Siemens Medical Solutions, Erlangen, Germany) in order to automatically calculate the flow rates and flow volumes.

Statistical analysis, including non-parametric sign tests, regression analysis, and t-tests, was performed to compare the flow data from the segmented acquisitions with the flow data from the non-segmented acquisitions. Most of the statistics was performed using Minitab (Version 13, Minitab, Inc., State College, PA).

\section{RESULTS}

Figure 1 shows representative magnitude and phase images from the non-seg and seg-9 PVM acquisitions from one subject at peak systole. Both sets of images are of sufficient quality for reliable analysis and flow quantification. Similar image quality was observed in all subjects for all three sequences used. 

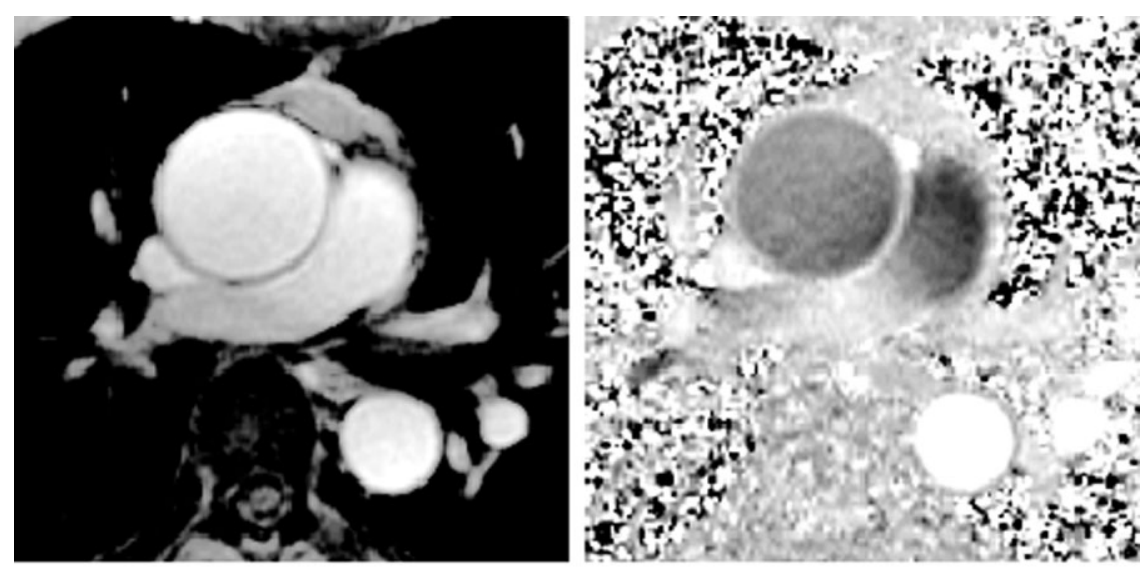

Figure 1. Magnitude (left) and phase (right) images acquired in the same subject using the non-segmented sequence (top) and the segmented sequence with nine lines per segment (bottom). Both sets of images are of sufficient quality for flow quantification analysis.
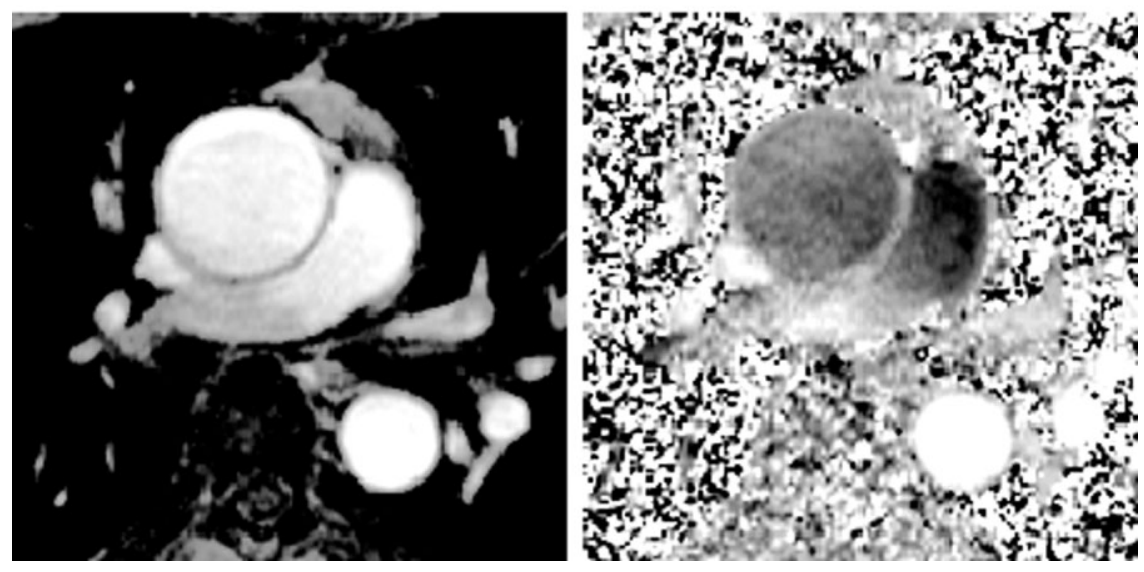

As expected, the seg-7 acquisitions provided higher temporal resolution than the seg-9 acquisitions (13-25 time-phases for seg-7 vs. 11-21 time-phases for seg-9); however, seg-9 provided greater scanning speed (13-16 seconds for seg-7 vs. 10-13 seconds for seg-9).

The flow curves obtained using the segmented acquisitions agreed with the curves obtained using the nonsegmented acquisitions, as seen in Figure 2 for four representative cases. In patients with aortic regurgitation, the flow curves showed diastolic reverse flow activity, as expected (e.g., subject 17, Fig. 2). Table 1 shows all of the flow volume results.

The results for the systolic and the total flow volumes from the segmented PVM acquisitions showed a close correlation (Pearson coefficients $\geq 0.94$, Table 2) and agreement (mean difference $<5 \%$ ) with those from the non-segmented acquisitions. Sign tests showed no statistically significant differences $(P$-values $>0.05)$ between the segmented and the non-segmented PVM measurements (Table 2). Figures 3 and 4 show the regression analysis results from the comparison between the segmented and the non-segmented systolic and total flow volumes, respectively. In all cases, the regression lines have slopes not significantly different from 1.0, and intercepts not significantly different from zero (as statistically determined via t-tests with $P$-values $>0.05)$. In addition, the coefficients of determination are large $\left(r^{2}>0.88\right)$, and the standard errors small (Table 2). Good correlation (Pearson coefficient $\geq 0.97$, Table 2) and no statistically significant differences were also found between the seg-7 and seg-9 flow volume results (refer to the regression analysis and sign test results in Fig. 5 and Table 2).

\section{DISCUSSION}

Non-segmented MR PVM is an accurate and precise velocimetric and flow quantification technique, and has been used clinically to measure blood flow and characterize blood flow patterns throughout the cardiovascular system. Despite its advantages, it is relatively slow, requiring several minutes for acquisition of a single velocity component in a slice. With the development of segmented $\mathrm{k}$-space acquisition schemes, rapid PVM has become available.

We recently evaluated the in vitro accuracy of segmented PVM with five, seven, and nine lines per segment under a variety of flow conditions (31). The results showed that segmented k-space PVM has high potential for blood flow quantification. Based on the positive in vitro results, the aim of the present study was to clinically evaluate the potential of segmented PVM to quantify blood flow in the aorta of human subjects. We did not evaluate the segmented scheme with five lines per segment clinically, because it would generally require more than 20 seconds per acquisition. A breath-hold of such duration may be difficult to achieve for many patients.

Due to the lack of a universally acceptable goldstandard blood flow quantification technique, in this study we considered non-segmented PVM as the reference technique. This can be justified by the large 

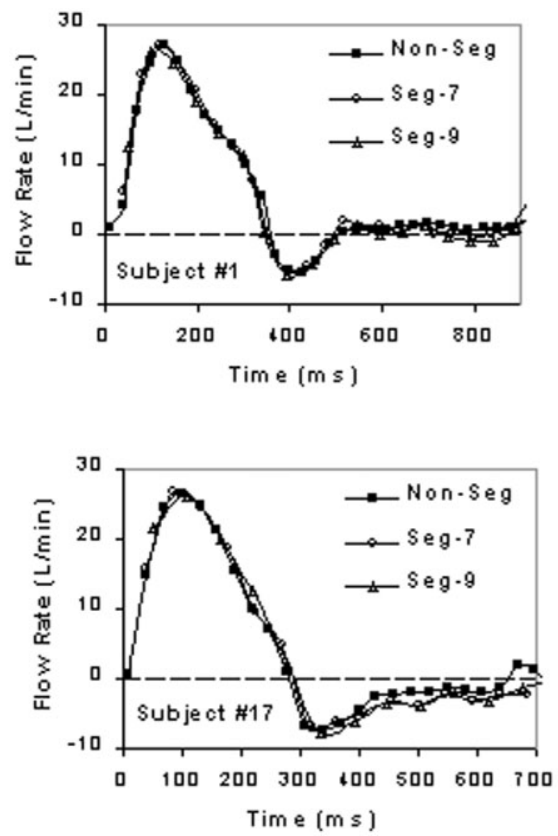
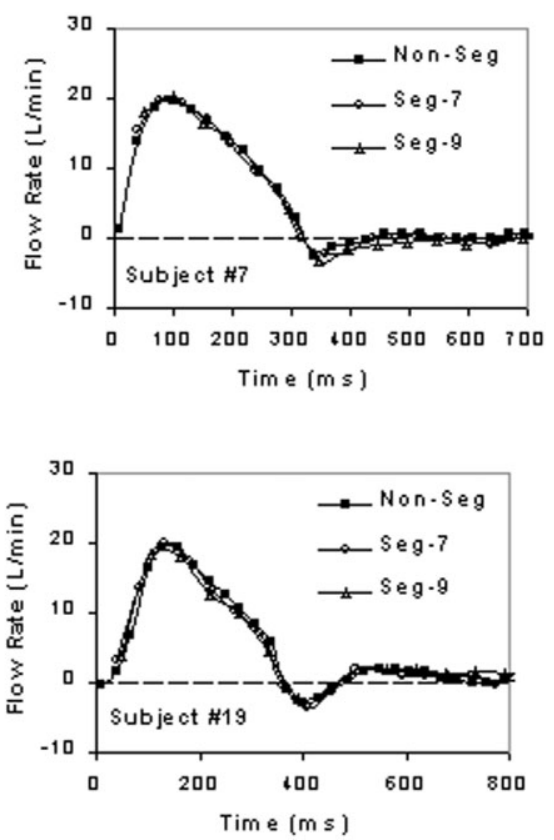

Figure 2. Measured aortic flow waveforms in four subjects using the non-segmented sequence, and two segmented sequences with seven and nine k-space lines per segment. A good agreement can be observed in all cases between the three sequences. number of previous evaluations of the non-segmented technique both in vitro and in vivo. Therefore, a comparison between segmented and non-segmented k-space PVM can provide important information about the reliability of segmented PVM as a flow quantification technique.

Image quality was good for all sequences used, facilitating image analysis and processing. The aortic crosssection could easily be identified for further analysis. Although the in-plane spatial resolution $\left(\cong 1-3 \mathrm{~mm}^{2}\right)$ was adequate to obtain the details of the velocity profile for a large vessel such as the ascending aorta, an even higher resolution (however, at the expense of scanning time) would be required for smaller vessels, as previously observed in vitro (31). Higher spatial resolution would also better resolve the velocity gradients immediately adjacent to the vessel wall (important to accurately calculate wall shear stress). However, the effects of reducing voxel size on the signal-to-noise ratio and, thus, image quality in vivo need to be thoroughly investigated.

The measured aortic flow curves in all subjects were very similar for all three sequences (non-seg, seg-7, and seg-9). It should be noted that, in seg-

Table 1

Flow Volume Results

\begin{tabular}{|c|c|c|c|c|c|c|}
\hline \multirow{2}{*}{ Subject no. } & \multicolumn{3}{|c|}{ Systolic volume, $\mathrm{ml} / \mathrm{cycle}$} & \multicolumn{3}{|c|}{ Total volume, $\mathrm{ml} /$ cycle } \\
\hline & Non-Seg & Seg-7 & Seg-9 & Non-Seg & Seg-7 & Seg-9 \\
\hline 1 & 90.1 & 91.7 & 90.3 & 88.1 & 90.8 & 86.1 \\
\hline 2 & 41.8 & 41.1 & 42.9 & 36.6 & 39.2 & 36.0 \\
\hline 3 & 55.8 & 58.4 & 59.4 & 36.8 & 37.2 & 33.7 \\
\hline 4 & 67.8 & 67.3 & 69.4 & 64.6 & 60.2 & 66.8 \\
\hline 5 & 57.5 & 61.9 & 58.9 & 57.0 & 58.8 & 58.3 \\
\hline 6 & 45.3 & 49 & 47.9 & 44.2 & 50.1 & 54.2 \\
\hline 7 & 67.9 & 67.9 & 68.9 & 67.1 & 64.1 & 62.6 \\
\hline 8 & 57.9 & 59.4 & 57.3 & 59.1 & 43.9 & 48.7 \\
\hline 9 & 52.4 & 49.1 & 48.5 & 48.4 & 47.8 & 46.4 \\
\hline 10 & 54.8 & 55.9 & 57 & 53.7 & 52.7 & 53.3 \\
\hline 11 & 63.8 & 63.7 & 64.3 & 58.5 & 57.4 & 61.0 \\
\hline 12 & 72.4 & 66.1 & 69.6 & 77.7 & 76.1 & 82.4 \\
\hline 13 & 68 & 64 & 67.2 & 60.5 & 67.1 & 68.4 \\
\hline 14 & 37.2 & 40.2 & 37.2 & 40.7 & 36.1 & 42.7 \\
\hline 15 & 48.5 & 48.3 & 47.3 & 39.9 & 36.6 & 36.3 \\
\hline 16 & 71.9 & 70.9 & 73.9 & 69.8 & 67.3 & 73.2 \\
\hline 17 & 72.2 & 77 & 78.3 & 53.9 & 50.5 & 51.8 \\
\hline 18 & 72.6 & 67.8 & 74.2 & 53.5 & 56.8 & 56.5 \\
\hline 19 & 66.2 & 65.1 & 64 & 68.0 & 70.2 & 71.4 \\
\hline 20 & 70.5 & 75.8 & 72.3 & 53.8 & 58.6 & 57.2 \\
\hline
\end{tabular}

Non-Seg = non-segmented; Seg-7 = segmented with seven lines per segment; Seg-9 = segmented with nine lines per segment. 
Table 2

Regression Analysis and Other Statistics

\begin{tabular}{|c|c|c|c|c|c|c|c|}
\hline $\begin{array}{c}\mathrm{Y} \\
\text { (mL/second) }\end{array}$ & $\begin{array}{c}\mathrm{X} \\
\text { (mL/second) }\end{array}$ & $\begin{array}{l}\text { Regression } \\
\text { equation }\end{array}$ & $\mathrm{R}^{2}$ & $\begin{array}{c}\text { SE } \\
\text { (mL/second) }\end{array}$ & $\begin{array}{l}\text { Mean \% } \\
\text { difference }\end{array}$ & $\begin{array}{l}\text { Sign test } \\
P \text {-value }\end{array}$ & $\begin{array}{c}\text { Pearson } \\
\text { correlation } \\
\text { coefficient }\end{array}$ \\
\hline \multicolumn{8}{|c|}{ Systolic flow volume } \\
\hline Seg-7 & Non-Seg & $Y=0.95 X+3.38$ & 0.937 & 3.2 & 0.8 & 1.0 & 0.97 \\
\hline Seg-9 & Non-Seg & $Y=1.01 X-0.14$ & 0.970 & 2.3 & 1.1 & 0.2 & 0.99 \\
\hline Seg-9 & Seg-7 & $Y=1.03 X-1.40$ & 0.964 & 2.6 & 0.5 & 0.8 & 0.98 \\
\hline \multicolumn{8}{|l|}{ Total flow volume } \\
\hline Seg-7 & Non-Seg & $Y=0.99 X+0.26$ & 0.885 & 5.0 & -0.8 & 0.8 & 0.94 \\
\hline Seg-9 & Non-Seg & $Y=1.02 X-0.57$ & 0.903 & 4.7 & 1.2 & 0.8 & 0.95 \\
\hline Seg-9 & Seg-7 & $Y=1.00 X+1.44$ & 0.942 & 3.6 & 2.3 & 0.8 & 0.97 \\
\hline
\end{tabular}

SE = standard error; Seg-7 = segmented with seven lines per segment; Seg-9 = segmented with nine lines per segment; Non-Seg=nonsegmented.

mented PVM with $N$ lines per segment (where $N$ is an odd number), the most influential information is contained in the central line of the segment, $(N+1) / 2$, because this is the closest line of the segment to the center of $\mathrm{k}$-space. Therefore, the time point corresponding to each cardiac phase was adjusted to be the time of acquisition of the central $\mathrm{k}$-space line of the segment. As a result, the segmented techniques cannot acquire data at the very beginning of the cardiac cycle when they are prospectively ECG-gated (in this study, the first time phase in the cycle corresponded to 8,40 , and $50 \mathrm{msec}$ after the QRS wave, for the non-seg, seg7 , and seg-9 acquisitions, respectively). This may cause uncertainties in measurements for patients with very rapid early systolic flow acceleration. Use of retrospective ECG-gating should eliminate this problem.

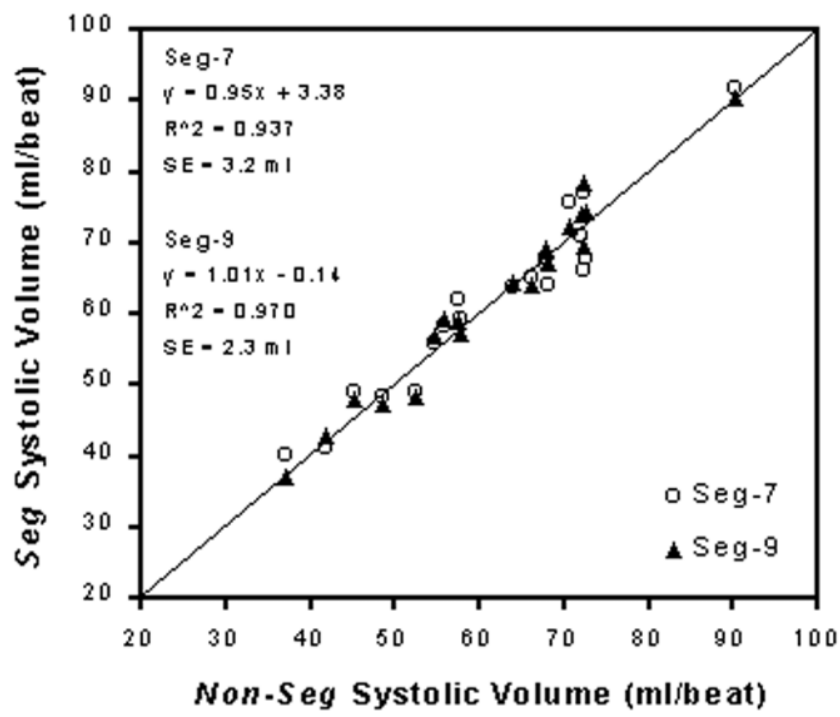

Figure 3. Comparison between the systolic flow volumes measured with the non-segmented and the two segmented sequences (non-Seg: non-segmented acquisition; Seg-7: segmented acquisition with seven $\mathrm{k}$-space lines per segment; Seg-9: segmented acquisition with nine k-space lines per segment). The diagonal line is the line of identity.
The seg-7 and seg-9 flow volume (systolic and total) results showed strong correlation and agreement with the non-seg flow volume results, suggesting that reliable blood flow quantification is possible using ultra-fast segmented PVM. Increasing the number of $\mathrm{k}$-space lines per segment to nine did not adversely affect the ability to sufficiently resolve the flow waveforms. The seg-9 scans were faster than the seg-7 scans by approximately three seconds, and provided acquisitions with good temporal resolution ( $>10$ time phases per cycle). Increasing the number of $\mathrm{k}$-space lines per segment above nine will certainly shorten the acquisition even more, but at the expense of temporal resolution. Reducing the temporal resolution may cause uncertainties in determining local velocity values such as peak systolic velocities. Therefore, the number of lines should be further increased only

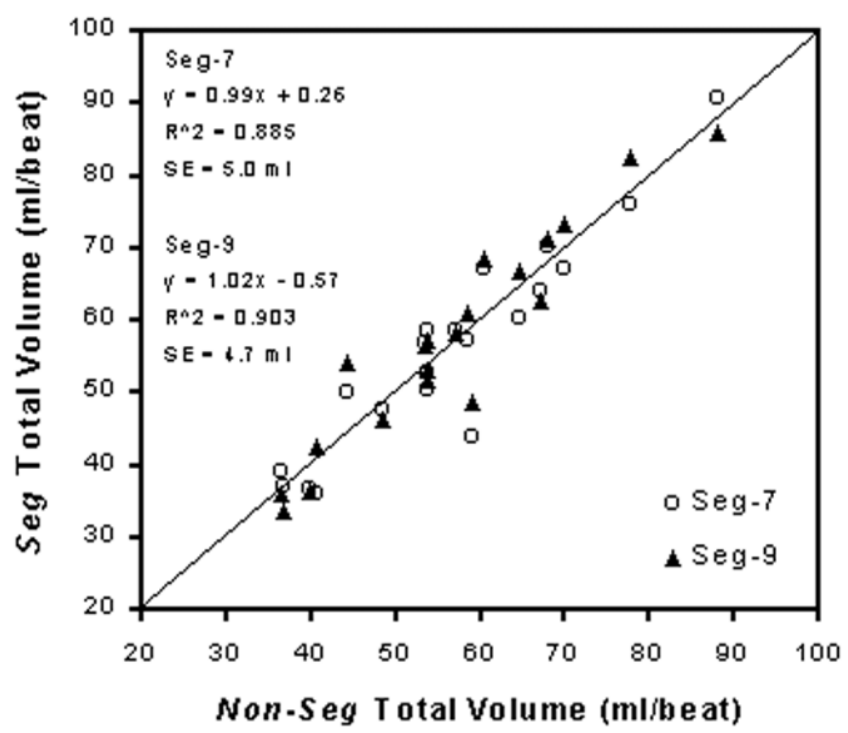

Figure 4. Comparison between the total flow volumes measured with the non-segmented and the two segmented sequences (non-Seg: non-segmented acquisition; Seg-7: segmented acquisition with seven $\mathrm{k}$-space lines per segment; Seg-9: segmented acquisition with nine k-space lines per segment). The diagonal line is the line of identity. 


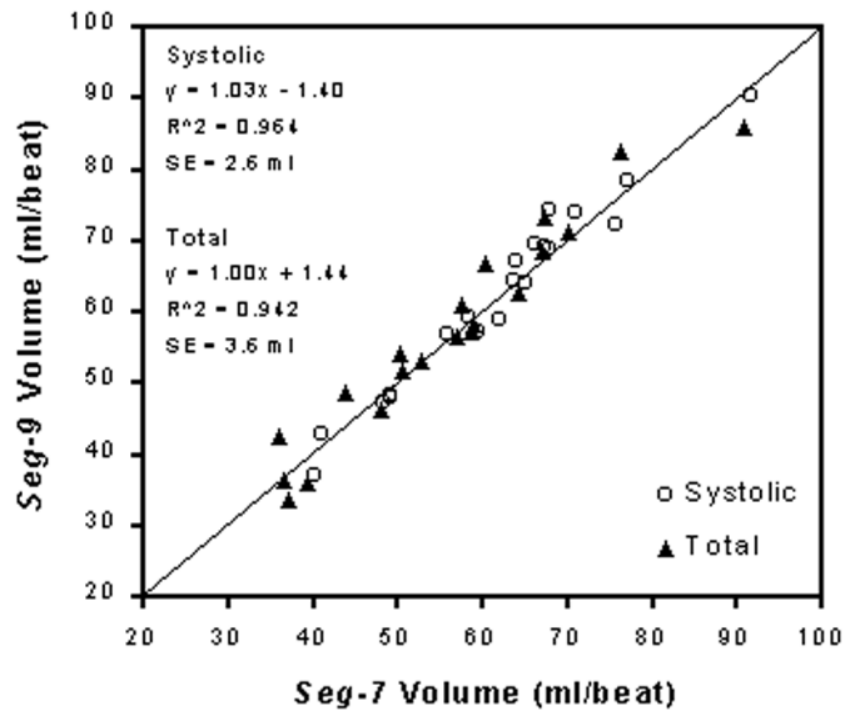

Figure 5. Comparison between the flow volumes (systolic and total) measured with the two segmented sequences (Seg-7: segmented acquisition with seven k-space lines per segment; Seg-9: segmented acquisition with nine k-space lines per segment). The diagonal line is the line of identity.

after assuring acquisition of an adequate number of time phases per cycle to prevent loss of valuable information and evaluating the acquisition technique in vitro and in vivo.

In conclusion, segmented $\mathrm{k}$-space magnetic resonance phase velocity mapping in the human ascending aorta produced flow results that agreed with those from non-segmented acquisitions. Increasing the number of lines per segment to nine allowed very fast acquisitions $(<15$ seconds) with adequate temporal resolution (less than $60 \mathrm{msec}$ between successive time phases). Segmented k-space MRPVM shows great clinical potential in blood flow quantification.

\section{REFERENCES}

1. Moran PR. A flow velocity zeugmatographic interlace for NMR imaging in humans. Magn Reson Imaging 1982;1:197-203.

2. Bryant DJ, Payne JA, Firmin DN, Longmore DB. Measurement of flow with NMR imaging using a gradient pulse and phase difference technique. J Comput Assist Tomogr 1984;8: 588-593.

3. Duerk JL, Pattanu PM. In-plane flow velocity quantification along the phase encoding axis in MRI. Magn Reson Imaging 1988;6:321333.

4. Meier D, Maier S, Bosiger P. Quantitative flow measurements on phantoms and on blood vessels with MR. Magn Reson Med 1988; 8:25-34.

5. Chatzimavroudis GP, Walker PG, Oshinski JN, Franch RH, Pettigrew RI, Yoganathan AP. Slice location dependence of aortic regurgitation measurements with MR phase velocity mapping. Magn Reson Med 1997;37:545-551.

6. Chatzimavroudis GP, Oshinski JN, Pettigrew RI, Walker PG, Franch RH, Yoganathan AP. Quantification of mitral regurgitation with MR phase-velocity mapping using a control volume method. J Magn Reson Imaging 1998;8:577-582.
7. Chatzimavroudis GP, Oshinski JN, Franch RH, Walker PG, Yoganathan AP, Pettigrew RI. Evaluation of the precision of magnetic resonance phase velocity mapping for blood flow measurements. J Cardiovasc Magn Reson 2001;3:11-19.

8. Dulce M-C, Mostbeck GH, O'Sullivan M, Cheitlin M, Caputo GR, Higgins CB. Severity of aortic regurgitation: interstudy reproducibility of measurements with velocity-encoded cine MR imaging. Radiology 1992;185:235-240.

9. Pettigrew RI, Dannels W, Galloway JR, et al. Quantitative phaseflow MR imaging in dogs by using standard sequences: comparison with in vivo flow-meter measurements. Am J Roentgenol 1987;148: 411-414.

10. Mohiaddin RH, Wann SL, Underwood R, Firmin DN, Rees S, Longmore DB. Vena caval flow: assessment with cine MR velocity mapping. Radiology 1990;177:537-541.

11. Pelc LR, Pelc NJ, Rayhill SC, et al. Arterial and venous blood flow: noninvasive quantitation with MR imaging. Radiology 1992;185: 809-812.

12. Underwood SR, Firmin DN, Klipstein RH, Rees RSO, Longmore DB. Magnetic resonance velocity mapping: clinical application of a new technique. Br Heart J 1987;57:404-412.

13. Klipstein RH, Firmin DN, Underwood SR, Rees RSO, Longmore DB. Blood flow patterns in the human aorta studied by magnetic resonance. Br Heart J 1987;58:316-323.

14. Kondo C, Caputo GR, Semelka R, Foster E, Shimakawa A, Higgins CB. Right and left ventricular stroke volume measurements with velocity-encoded cine MR imaging: in-vitro and in-vivo validation. AJR Am J Roentgenol 1991;157:9-16.

15. Kilner PJ, Yang GZ, Mohiaddin RH, Firmin DN, Longmore DB. Helical and retrograde secondary flow patterns in the aortic arch studied by three-directional magnetic resonance velocity mapping. Circulation 1993;88:2235-2247.

16. Bogren HG, Buonocore MH. Blood flow measurements in the aorta and major arteries with MR velocity mapping. J Magn Reson Imaging 1994;4:119-130.

17. Chatzimavroudis GP, Oshinski JN, Franch RH, Pettigrew RI Walker PG, Yoganathan AP. Quantification of the aortic regurgitant volume with magnetic resonance phase velocity mapping: a clinical investigation of the importance of imaging slice location. J Heart Valve Dis 1998;7:94-101.

18. Walker PG, Oyre S, Pedersen EM, Houlind K, Guenet FSA, Yoganathan AP. A new control volume method for calculating valvular regurgitation. Circulation 1995;92:579-586.

19. Walker PG, Houlind K, Djurhuus C, Kim WY, Pedersen EM. Motion correction for the quantification of mitral regurgitation using the control volume method. Magn Reson Med 2000;43: 726-733.

20. Firmin DN, Klipstein RH, Hounsfield GL, Paley MP, Longmore DB. Echo-planar high-resolution flow velocity mapping. Magn Reson Med 1989;12:316-327.

21. McKinnon GC, Debatin JF, Wetter DR, von Schulthess GK. Interleaved echo planar flow quantitation. Magn Reson Med 1994;32: 263-267.

22. Mohiaddin RH, Gatehouse PD, Firmin DN. Exercise-related changes in aortic flow measured with spiral echo-planar MR velocity mapping. J Magn Reson Imaging 1995;5:159163.

23. Thomsen C, Cortsen M, Söndergaard L, Henriksen O, Sthlberg F, A segmented k-space velocity mapping protocol for quantification of renal artery blood flow during breath-holding. J Magn Reson Imaging 1995;5:393-401.

24. Debatin JF, Leung DA, Wildermuth S, Botnar R, Felblinger J, McKinnon GC. Flow quantitation with echo-planar phase-contrast velocity mapping: in vitro and in vivo evaluation. J Magn Reson Imaging 1995;5:656-662.

25. Chuang ML, Chen MH, Khasgiwala VC, McConnell MV, Edelman RR, Manning WJ. Adaptive correction of imaging plane position in segmented k-space cine cardiac MRI. J Magn Reson Imaging 1997; 7:811-814.

26. Davis CP, Liu P-F, Hauser M, Göhde SC, von Schulthess GK, Debatin JF. Coronary flow and coronary flow reserve measurements in humans with breath-held magnetic resonance phase contrast velocity mapping. Magn Reson Med 1997;37: 537-544. 
27. Bock M, Schoenberg SO, Schad LR, Knopp MV, Essig M, van Kaick G. Interleaved gradient-echo planar (IGEPI) and phase contrast CINE-PC flow measurements in the renal artery. J Magn Reson Imaging 1998;8:889-895.

28. Poutanen V-P, Kivisaari R, Häkkinen A-M, Savolainen S, Hekali P, Standertskjöld-Nordenstam C-G. Multiphase segmented k-space velocity mapping in pulsatile flow waveforms. Magn Reson Imaging 1998; 16:261-270.

29. Laffon E, Lecesne R, de Ledinghen V, et al. Segmented 5 versus nonsegmented flow quantitation: comparison of portal vein flow measurements. Invest Radiol 1999;34:176-180.

30. Nagel E, Bornstedt A, Hug J, Schnackenburg B, Wellnhofer E, Fleck E. Noninvasive determination of coronary blood flow velocity with magnetic resonance imaging: comparison of breath-hold and navigator techniques with intravascular ultrasound. Magn Reson Med 1999;41:544-549.

31. Zhang H, Halliburton SS, Moore JR, et al. Ultra-fast flow quantification with segmented k-space magnetic resonance phase velocity mapping. Ann Biomed Eng 2002;30:120-128.

32. Markl M and Hennig J. Phase contrast MRI with improved temporal resolution by view sharing: $k$-space related velocity mapping properties. Magn Reson Imaging 2001;19:669-676.

33. Walker PG, Cranney GB, Scheidegger MB, Waseleski G, Pohost GM, Yoganathan AP. Semiautomated method for noise reduction and background phase error correction in MR phase velocity data. J Magn Reson Imaging 1993;3:521-530. 